\section{Clostridium difficile: la belle et la bête}

Lucie Etienne-Mesmin
Center for Inflammation Immunity and Infection, Institute for Biomedical Sciences, Georgia State University, 100 Piedmont Ave SE, 30303 Atlanta, Georgie, États-Unis. lucie.etiennemesmin@yahoo.fr

5 antibiotiques administrés dans l'eau de boisson ad libitum simultanément avec une injection intra-péritonéale d'un autre antibiotique) avant infection par des spores de la souche de référence C. difficile VPI 10463 (souche virulente ATCC 43255, produisant les toxines TcdA et $T c d B$ ). La présence de $C$. difficile dans les fèces des souris infectées a été analysée par la quantification du gène $t c d A$, codant la toxine TcdA, par PCR (polymerase chain reaction) quantitative : cette méthode fondée sur l'extraction d'ADN à partir de matériel fécal permet de s'affranchir de l'utilisation des techniques de cultures bactériennes anaérobies classiques [10].

\section{Identification et séquençage d'une} nouvelle souche de Clostridium difficile murine protégeant contre les infections par une souche pathogène Après le traitement par antibiotiques, nous avons observé un pourcentage important de souris non infectées par $C$. difficile VPI 10463 qui présentait néanmoins des niveaux importants du gène $\operatorname{tcd} A$ dans leurs fèces, sans pour autant développer des signes cliniques de pathologies intestinales. Le transfert du microbiote fécal de ces souris conventionnelles traitées par antibiothérapie et détectées positives pour tcdA à des souris axéniques n'induit pas de signes cliniques chez les souris receveuses, indiquant que la souche de C. difficile présente chez les souris conventionnelles n'est pas virulente. Ces résultats montrent donc que l'antibiothérapie induit des modifications du microbiote intestinal qui permettent l'expansion d'une souche endogène de $C$. difficile 


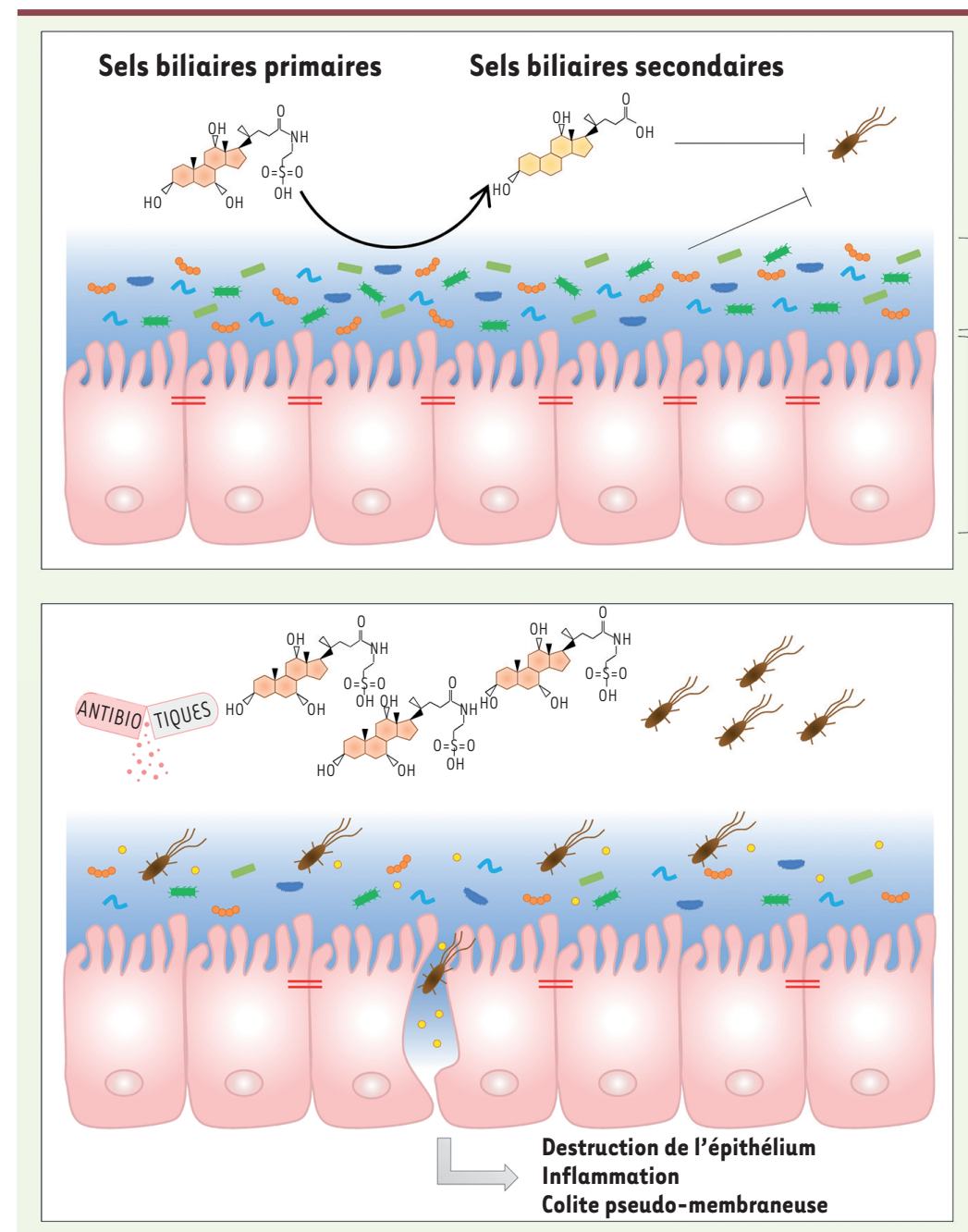
a. Homéostasie
Les sels biliaires primaires sont convertis en sels biliaires secondaires et inhibent la multiplication de $C$. difficile (inhibition de la germination)

\section{Mucus}

\section{Épithélium intestinal}

\section{b. Dysbiose du microbiote après traitement antibiotique et infection à $C$. difficile}

- Les sels biliaires primaires ne sont plus convertis en sels biliaires secondaires et n'inhibent plus la multiplication de C. difficile - C. difficile produit des toxines qui vont détruire les jonctions serrées et induire une réponse inflammatoire (recrutement de polynucléaires), conduisant à une colite pseudo-membraneuse

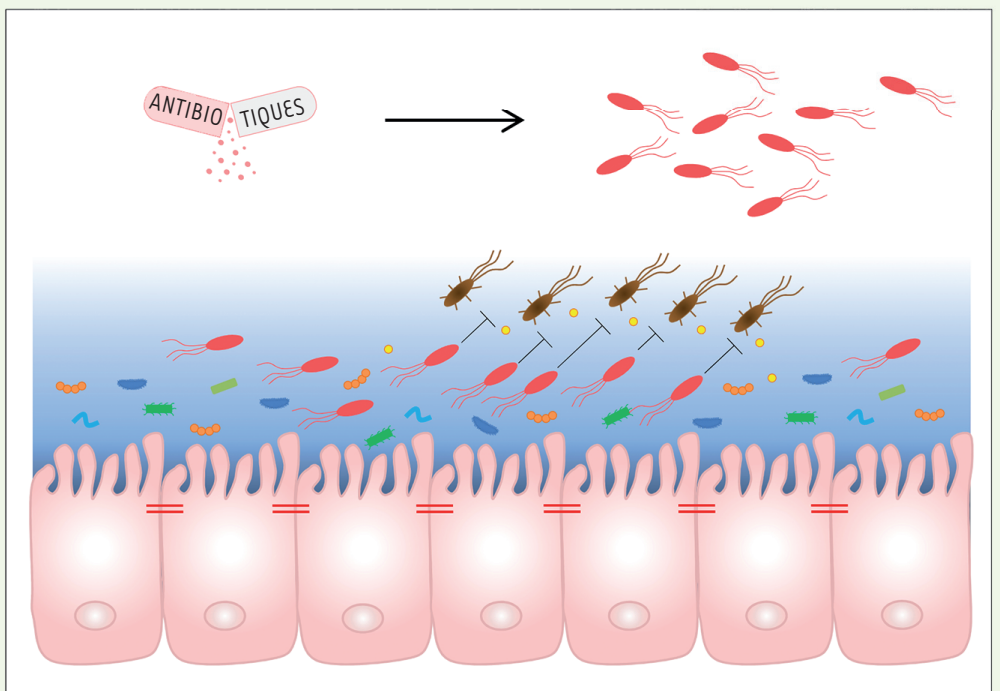

\section{c. Dysbiose du microbiote après traitement antibiotique et apparition d'une souche non pathogène de $C$. difficile}

- Apparition de $C$. difficile LEMI

- C. difficile VPI 10463 ne peut plus coloniser le tractus digestif

Mécanismes

- Exclusion du pathogène (compétition pour les nutriments, etc.)

- Antagonisme direct

- Immunomodulation (défensines, sécrétion de molécules anti-inflammatoires, etc.) - Inhibition des facteurs de virulence

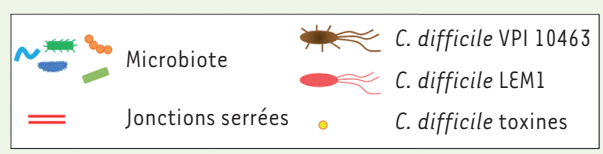

Figure 1. Rôle du microbiote dans la défense contre Clostridium difficile. A. Lorsque le microbiote est intact, certaines bactéries convertissent les sels biliaires primaires en sels biliaires secondaires s'opposant ainsi à la multiplication et à la colonisation par $C$. difficile. B. Lorsque le microbiote est perturbé suite à un traitement antibiotique, les sels biliaires primaires ne sont plus convertis en sels biliaires secondaires et ne peuvent plus exercer leur action inhibitrice sur $C$. difficile. $C$. Lorsque le microbiote est perturbé par un traitement antibiotique, la souche non pathogène $C$. difficile LEMI apparaît et empêche la colonisation par la souche pathogène C. difficile VPI 10463. 
qui, bien que produisant des toxines, reste avirulente [10].

Une analyse rétrospective du microbiote intestinal des souris conventionelles exposées au traitement antibiotique a permis de mettre en évidence des altérations majeures de la composition et de la diversité des populations microbiennes, à l'origine de susceptibilités différentes à $C$. difficile et définissant deux catégories d'animaux: des animaux dits «résistants » et des animaux dits «sensibles». En effet, les souris considérées comme résistantes présentaient un microbiote constitué d'une faible abondance d'une souche endogène de $C$. difficile, nommée LEMI, capable de produire des toxines mais n'induisant pas de signes cliniques. À l'opposé, chez les animaux sensibles, qui succombaient en quelques jours à une infection par la souche virulente $C$. difficile VPI 10463, la souche LEMI n'était pas détectée.

La souche murine de C. difficile LEMI a été isolée du microbiote des animaux « résistants» par des techniques de bactériologie classique (isolement sur milieu gélosé sélectif suivi d'une culture en chambre anaérobie), et a été caractérisée par une amplification PCR des ARN ribosomaux (ou ribotypage : $P C R$ ribotyping, en anglais). L'identification du profil obtenu par ribotypage et sa comparaison aux profils des souches de références ont révélé que la souche LEMI n'avait pas été identifiée à ce jour. L'utilisation d'une technique très sensible de PCR nichée (nested-PCR en anglais) a montré que $40 \%$ des souris résistantes possédaient, dans leur microbiote, la souche $C$. difficile LEMI avant même le traitement antibiotique alors qu'aucune des souris «susceptibles» ne la présentait, suggérant que certains animaux hébergeaient naturellement cette souche dans leur microbiote et

\footnotetext{
${ }^{1}$ La technique de nested-PCR (PCR nichée) se pratique comme la PCR (polymerase chain reaction) avec un couple d'amorces externes pour quelques cycles d'amplification. Ensuite, un excès d'une amorce interne est ajouté avant de prolonger l'amplification.
}

ce avant même l'exposition au traitement antibiotique. Le génome de la souche $C$. difficile LEMI a été séquencé et a été comparé à celui de la souche de référence $C$. difficile VPI 10463 [11]. L'alignement des deux génomes a permis de mettre en évidence de nombreuses translocations et des réarrangements génomiques avec, cependant, un degré global de similarité élevé (95\%) entre les deux souches.

Cette étude montre que l'administration exogène de la souche $C$. difficile LEMI n'induit pas de signes pathologiques dans le modèle de souris conventionelles recevant une antibiothérapie, $\mathrm{ni}$ chez les souris axéniques (souris pourtant sujettes à développer des signes cliniques fatals en quelques heures après une infection par la souche pathogène $C$. difficile VPI 10463). La faible virulence de $C$. difficile LEMI, même lorsqu'elle est administrée à fortes doses, peut être expliquée par sa faible capacité à produire les toxines par rapport à $C$. difficile VPI 10463. Nos résultats ont également démontré que l'administration préventive, ou concomitante, de la souche $C$ difficile $L E M I$ avec $C$. difficile VPI 10463, permet de protéger les animaux contre une infection qui est normalement létale. La souche $C$. difficile LEMI est en effet capable de diminuer la colonisation intestinale par la souche VPI 10463, et donc les signes pathologiques qui sont associés à l'infection, chez des souris conventionnelles et des souris axéniques, probablement par un mécanisme de compétition vis-à-vis d'une niche écologique commune [10] (Figure 1). La capacité de la souche C. difficile LEMI à protéger contre une souche virulente corrobore les observations démontrant que des souches non-toxinogènes peuvent prévenir la mortalité induite par $C$. difficile chez les hamsters [12] et réduire le taux de récidive chez l'homme [13].

\section{Conclusions et perspectives}

Les résultats que nous avons obtenus mettent en évidence la présence, au niveau du microbiote intestinal murin, d'une bactérie à potentiel bénéfique. Ils suggèrent son possible usage thérapeutique pour le traitement des ICD. Les implications de cette étude sont nombreuses mais la compréhension des mécanismes de protection de la souche C. difficile LEMI vis-à-vis de C. difficile VPI 10463 reste à élucider. La présence de bactériophages et/ou la sécrétion de molécules inhibitrices produites par la souche $C$. difficile LEMI et pouvant agir sur C. difficile VPI 10463 sont des pistes envisagées. L'extrapolation de ces résultats chez l'homme suggèrent que certaines ICD asymptomatiques, qui se manifestent dans les hôpitaux, pourraient être en fait dues à des souches de $C$. difficile présentes à de très faibles niveaux (voire indétectables) dans le microbiote intestinal et non à l'acquisition de souches de $C$. difficile présentes dans l'environnement hospitalier.

Le traitement antibiotique induit de nombreuses perturbations du microbiote. Certaines des espèces bactériennes sont relativement résistantes. Nous recherchons, par l'analyse des données de séquençage et par des techniques de culture anaérobie, à définir des taxons ${ }^{2}$ bactériens dont l'élimination, par le traitement antibiotique, serait corrélée avec la prolifération de la souche $C$. difficile LEMl endogène et pourraient représenter de nouvelles voies thérapeutiques pour le traitement des ICD. $\diamond$

Clostridium difficile: the beauty and the beast

\section{LIENS D'INTÉRÊT}

Les auteurs déclarent n'avoir aucun lien d'intérêt concernant les données publiées dans cet article.

\section{REMERCIEMENTS}

Je remercie Benoit Chassaing (Institute for biomedical sciences, Atlanta, GA, États-Unis) pour la relecture critique du manuscrit.

\footnotetext{
${ }^{2}$ Un taxon correspond à une entité d'êtres vivants regroupés parce qu'ils possèdent des caractères en communs du fait de leur parenté.
} 


\section{RÉFÉRENCES}

1. Eckert C, Barbut F. Infections associées à Clostridium difficile. Med Sci (Paris) 2010 ; $26: 153-8$.

2. Bouza $\varepsilon$. Consequences of Clostridium difficile infection: understanding the healthcare burden. Clin Microbiol Infect 2012; 18 (suppl 6) : 5-12.

3. Davies KA, Longshaw CM, Davis GL, et al. Underdiagnosis of Clostridium difficile across Europe: the European, multicentre, prospective, biannual, point-prevalence study of Clostridium difficile infection in hospitalised patients with diarrhoea (EUCLID). Lancet Infect Dis 2014 ; 14 : 1208-19.

4. Lessa FC, Mu Y, Bamberg WM, et al. Burden of Clostridium difficile infection in the United States. N Engl J Med 2015 ; 372 : 825-34.

5. Martin JS, Monaghan TM, Wilcox MH. Clostridium difficile infection: epidemiology, diagnosis and understanding transmission. Nat Rev Gastroenterol Hepatol 2016; $13: 206-16$.

6. Buffie CG, Bucci V, Stein RR, et al. Precision microbiome reconstitution restores bile acid mediated resistance to Clostridium difficile. Nature $2015 ; 517: 205-8$

7. Rupnik M, Wilcox MH, Gerding DN. Clostridium difficile infection: new developments in epidemiology and pathogenesis. Nat Rev Microbiol 2009 ; 7 : 526-36.

8. Chen X, Katchar K, Goldsmith JD, et al. A mouse model of Clostridium difficile-associated disease. Gastroenterology 2008 ; 135 : 1984-92.

9. Reeves AE, Koenigsknecht MJ, Bergin IL, Young VB. Suppression of Clostridium difficile in the gastrointestinal tracts of germfree mice inoculated with a murine isolate from the family Lachnospiraceae. Infect Immun 2012 ; 80 : 3786-94.

10. Etienne-Mesmin L, Chassaing B, Adekunle 0 , et al. Toxin-positive Clostridium difficile latently infect mouse colonies and protect against highly-pathogenic C. difficile. Gut 2017 ; pii : gutjnl-2016-313510.

11. Etienne-Mesmin L, Chassaing B, Adekunle 0 , et al. Genome sequence of a toxin-positive Clostridium difficile strain isolated from murine feces. Genome Announc 2017 ; 5 : pii : e00088-17.

12. Nagaro KJ, Phillips ST, Cheknis AK, et al. Nontoxigenic Clostridium difficile protects hamsters against challenge with historic and epidemic strains of toxigenic BI/NAP1/027 C. difficile. Antimicrob Agents Chemother $2013 ; 57$ : 5266-70.

13. Gerding DN, Meyer T, Lee C, et al. Administration of spores of nontoxigenic Clostridium difficile strain M3 for prevention of recurrent $C$. difficile infection: a randomized clinical trial. JAMA 2015 ; 313 : 1719-27.

\section{NOUVELLE}

\section{Les gardiens de nos rêves identifiés}

\section{Une cible neuronale de la maladie de Parkinson?}

Patrice Fort ${ }^{1,2}$, Sara Valencia Garcia ${ }^{1-3}$

\author{
${ }^{1}$ SLEEP Team, Centre de recherche en neurosciences de Lyon \\ (CRNL), CNRS UMR 5292, Inserm U1028, 7, rue Guillaume \\ Paradin, F-69008 Lyon, France. \\ ${ }^{2}$ SLEદP Team, université Claude Bernard Lyon 1, \\ 7 , rue Guillaume Paradin, \\ 69372, Lyon, France. \\ ${ }^{3}$ Friedrich Miescher institute for biomedical research, \\ maulbeerstrasse 66 (R-1066.4.28), 4058, Bâle, Suisse. \\ patrice.fort@univ-lyonl.fr
}

> Dormir est une activité biologique commune au règne animal. Avec l'apparition des mammifères, est survenue une évolution majeure prenant la forme d'un second état de sommeil dit paradoxal (SP ou rapid eye movement [REM], sleep en anglais). Décrit seulement à la fin des années 1950, le sommeil paradoxal demeure aujourd'hui fascinant, en particulier pour sa concomitance avec l'activité oniriquel. Le sommeil paradoxal se caractérise par une activité cérébrale maximale, supérieure à celle de la veille, paradoxalement associée à une immobilité corporelle qui résulte d'une atonie de la musculature posturale empêchant tous mouvements intempestifs, potentiellement dangereux pour le rêveur coupé du monde extérieur. En 1986, une

${ }^{1}$ Qui a rapport au rêve.

pathologie (la parasomnie) consécutive à la perte totale ou intermittente de l'atonie musculaire pendant le sommeil paradoxal, a été décrite [1]. Le patient présentant cette pathologie, bien qu'endormi et inconscient, montre d'impressionnants mouvements complexes, involontaires, souvent violents et agressifs, qui s'accompagnent de vocalisations et de cris: le dormeur extérioriserait ses rêves $[2,3](\rightarrow)$.

$(\rightarrow)$ Voir la Nouvelle de J.F. Gagnon et al., $\mathrm{m} / \mathrm{s} \mathrm{n}{ }^{\circ} 10$ octobre 2009 page 782

Outre son impact délétère sur la qualité de vie des patients, des études longitudinales récentes ont établi que ces troubles comportementaux en sommeil paradoxal (TCSP, ou REM sleep behavior disorder [RBD]) constituent un marqueur diagnostique précoce (supérieur à 10 ans) de maladies neurodégénératives de type synucléinopathique ${ }^{2}$ (comme la maladie de Parkinson, la démence à corps de Lewy, l'atrophie multi-systématisée) [4, 5]. Selon l'hypothèse étiologique actuelle, le RBD reflèterait une atteinte synucléinopathique des neurones à l'origine de l'atonie musculaire pendant le sommeil paradoxal. En effet chez ces patients, la zone pontique cérébrolésée inclut le noyau sublatérodorsal (SLD) qui est constitué de neurones excitateurs glutamate actifs uniquement durant le sommeil paradoxal. Ils sont considérés comme générateurs de cet état de sommeil paradoxal en synchronisant l'ensemble de ses signes pathognomoniques (comme l'activation corticale,

\footnotetext{
${ }^{2}$ Les synucléinopathies sont un groupe de maladies neurodégénératives caractérisées par l'accumulation anormale d'agrégats d'alpha-synucléine dans les neurones, les fibres nerveuses ou les cellules gliales.
} 